\title{
In reply: Use of early postoperative serum creatinine elevation to identify acute kidney injury after cardiac surgery
}

\author{
Keyvan Karkouti, MD (1)
}

Received: 11 September 2017/Accepted: 20 September 2017/Published online: 25 September 2017

(C) Canadian Anesthesiologists' Society 2017

\section{To the Editor,}

Dr. Li et al. raise several important and interesting questions about our paper, ${ }^{1,2}$ and I appreciate the opportunity to address them. One question was whether we corrected postoperative serum creatinine measures based on the perioperative fluid balance. We did not do so because we have no confidence in the accuracy of the recorded perioperative fluid balance, which has been shown to be an unreliable measure of changes in body weight associated with cardiac surgery. ${ }^{3}$

Another question relates to our choice of the $30 \%$ early rise in creatinine (the ratio of the postoperative creatinine level to the preoperative creatinine level; $\Delta$ Creat $^{\text {ratio }}$ ) as the threshold for early diagnosis of acute kidney injury (AKI) because a smaller threshold of $10 \%$ is also predictive of AKI. ${ }^{4}$ Our $30 \%$ threshold provided the greatest proportion of correctly classified observations and maximized the positive predictive value (which was $69 \%$ for the $30 \%$ threshold versus $39 \%$ for the $10 \%$ threshold). Maximizing the positive predictive value is particularly desirable if $\Delta$ Creat $^{\text {ratio }}$ is to be used for early identification of patients to include in clinical trials aimed at ameliorating AKI.

Dr. Li et al. correctly state that, by externally validating the relation between $\Delta \mathrm{Creat}^{\text {ratio }}$ and AKI stage I only (i.e., $>1.5$-fold increase in serum creatinine) - the mildest AKI category of the consensus recommendations of the AKI Network classification ${ }^{5}$ - we neglected the more severe and clinically relevant categories of AKI. As there is a direct relation between the severity of AKI after cardiac surgery and adverse clinical outcomes, ${ }^{6}$ this point is an important shortcoming. We have now externally validated the relation between $\Delta \mathrm{Creat}^{\text {ratio }}$ and AKI stages II and III (representing a $>2.0$-fold and $>3.0$-fold increase in serum creatinine, respectively) (Table).

The final question relates to the predictive value of $\Delta$ Creat $^{\text {ratio }}$ relative to existing clinical prediction models,

Table Area under the receiver operating characteristic curve for the relation between $\Delta$ Creat $^{\text {ratio }}$ and the severity of acute kidney injury ${ }^{5}$

\begin{tabular}{lll}
\hline AKI definition & $\begin{array}{l}\text { Development dataset } \\
\text { AUROC (95\% CI) }\end{array}$ & $\begin{array}{l}\text { Validation dataset } \\
\text { AUROC (95\% CI) }\end{array}$ \\
\hline Stage I (> 1.5-fold increase in creatinine) & $0.78(0.75$ to 0.80$)$ & $0.77(0.75$ to 0.79$)$ \\
Stage II (> 2.0-fold increase in creatinine) & $0.81(0.77$ to 0.85$)$ & $0.85(0.81$ to 0.88$)$ \\
Stage III $(>3.0$-fold increase in creatinine) & $0.88(0.80$ to 0.97$)$ & $0.90(0.85$ to 0.95$)$ \\
\hline
\end{tabular}

$\mathrm{AKI}=$ acute kidney injury; AUROC $=$ area under the receiver operating characteristic (curve); $\mathrm{CI}=$ confidence interval

K. Karkouti, MD (凹)

Department of Anesthesia and Institute of Health Policy

Management and Evaluation, Peter Munk Cardiac Centre and the

Toronto General Research Institute, Toronto General Hospital,

University Health Network, University of Toronto, Toronto, ON,

Canada

e-mail: keyvan.karkouti@uhn.ca 
such as the Cleveland risk score. ${ }^{7}$ We measured the relative predictive values of $\Delta \mathrm{Creat}^{\text {ratio }}$ and the Cleveland risk score in the logistic regression model and found that $\Delta$ Creat ${ }^{\text {ratio }}$ had a substantially higher predictive value than the Cleveland risk score (Table 3 of the original paper). In addition, the area under the receiver operating characteristic (AUROC) curve of the model for AKI stage I that included only $\Delta$ Creat $^{\text {ratio }}$ was $0.78(95 \%$ confidence interval $[\mathrm{CI}], 0.75$ to 0.80$)$. Substituting $\Delta$ Creat $^{\text {ratio }}$ with the Cleveland risk score in the model reduces the AUROC to 0.64 (95\% CI, 0.61 to 0.67 ). It is important to note that the purpose of the Cleveland risk score is to predict renal replacement therapy, not AKI. Nevertheless, it is not surprising that a postoperative variable is more predictive than a score derived from preoperative variables.

Conflicts of interest None declared.

Editorial responsibility This submission was handled by Dr. Philip M. Jones, Associate Editor, Canadian Journal of Anesthesia.

\section{References}

1. Li HY, Xиe FS, Liu YY. Use of early postoperative serum creatinine elevation to identify acute kidney injury after cardiac surgery. Can J Anesth 2018; 65. DOI:10.1007/s12630-017-0979-9.

2. Karkouti K, Rao V, Chan CT. Wijeysundera DN; TACS Investigators. Early rise in postoperative creatinine for identification of acute kidney injury after cardiac surgery. Can. J Anesth 2017; 64: 801-9.

3. Eastwood GM. Evaluating the reliability of recorded fluid balance to approximate body weight change in patients undergoing cardiac surgery. Heart Lung 2006; 35: 27-33.

4. Ho J, Reslerova M, Gali B, et al. Serum creatinine measurement immediately after cardiac surgery and prediction of acute kidney injury. Am J Kidney Dis 2012; 59: 196-201.

5. Mehta RL, Kellum JA, Shah SV, et al. Acute Kidney Injury Network: report of an initiative to improve outcomes in acute kidney injury. Crit Care 2007; 11: R31.

6. Karkouti K, Wijeysundera DN, Yau TM, et al. Acute kidney injury after cardiac surgery: focus on modifiable risk factors. Circulation 2009; 119: 495-502.

7. Thakar CV, Arrigain S, Worley S, Yared JP, Paganini EP. A clinical score to predict acute renal failure after cardiac surgery. J Am Soc Nephrol 2005; 16: 162-8. 